\title{
Syndrome de Gougerot-Sjögren: manifestations buccales et prise en charge
}

\author{
Fouzia Hakkou*, Saliha Chbicheb, Wafae El Wady \\ Service d'Odontologie chirurgicale, CCTD, Rabat, Maroc
}

(Reçu le 12 août 2011, accepté le 3 octobre 2011)

\begin{abstract}
Mots clés : syndrome de GougerotSjögren / syndrome sec / xérostomie / kératoconjonctivite sèche / manifestations générales
\end{abstract}

Key words: Sjögren's syndrome / sicca syndrome / xerostomia / keratoconjunctivitis sicca / systemic manifestations

\begin{abstract}
Résumé - Le syndrome de Gougerot-Sjögren (SGS) est une affection auto-immune touchant en premier lieu les glandes exocrines. Sa principale manifestation clinique est le syndrome sec salivaire et/ou oculaire par infiltration lymphocytaire des glandes salivaires et/ou lacrymales. Le médecin dentiste est surtout consulté pour les complications bucco-dentaires et l'inconfort buccal engendrés par les variations qualitatives et quantitatives de la salive. Il a un rôle important à jouer dans le dépistage et le diagnostic précoce de cette affection. Il participe à la prévention des complications en motivant le patient pour l'hygiène bucco-dentaire et en surveillant l'évolution de la pathologie. Le traitement a pour objectif essentiel d'améliorer le confort du malade.
\end{abstract}

\begin{abstract}
Sjögren's syndrome: oral manifestations and management. Sjögren's syndrome is an immune disease affecting primarily the exocrine glands. The main clinical manifestation is salivary and/or ocular dryness due to a lymphocytic infiltration of the salivary or lacrimal glands or both. The dentist is usually consulted for the oral complications and the oral discomfort engendered by the qualitative and quantitative salivary changes. It has an important role to play in the screening and early diagnosis of this condition. He is involved in the prevention of complications by educating the patient about oral hygiene and monitoring the evolution of the pathology. The treatment will mainly aim to improve the comfort of the patient.
\end{abstract}

La première description du syndrome revient à MikuliczRadecki, chirurgien polonais, qui décrivit en 1892 un homme présentant une tuméfaction bilatérale des glandes parotides et des glandes lacrymales avec un infiltrat mononucléé [1]. Gougerot individualisa le syndrome en 1925 qui réunit toutes les sécheresses muqueuses. Il l'attribua à une atrophie glandulaire [2]. En 1933, l'ophtalmologue suédois Henrik Sjögren a décrit, en détail, les manifestations cliniques et les images histologiques retrouvées chez 19 femmes ayant une sécheresse buccale et oculaire. Sjögren a utilisé le terme de kératoconjonctivite sèche pour définir l'atteinte oculaire et la distinguer des yeux secs causés par une déficience en vitamine $\mathrm{A}[1,2]$.

Le syndrome de Gougerot-Sjögren (SGS) est une maladie auto-immune caractérisée par une dégénérescence progressive des glandes exocrines, aboutissant à une sécheresse des muqueuses et des conjonctives (syndrome sec). Histologiquement, ce syndrome est caractérisé par une infiltration lymphoïde des glandes exocrines, en particulier des glandes salivaires et lacrymales, et biologiquement par la production de nombreux autoanticorps (FAN, anti-SSA, anti-SSB, facteur rhumatoïde). Une vascularite peut être associée et l'infiltration lymphoïde intéresser d'autres organes. Si la maladie apparait isolée, il s'agit d'un SGS primaire. S'il existe une maladie auto-immune associée (polyarthrite rhumatoïde, lupus érythémateux disséminé...), on parle alors de SGS secondaire [3].

\section{Épidémiologie}

Le SGS primitif est la plus fréquente des connectivites après la polyarthrite rhumatoïde [2]. Sa prévalence est estimée entre 0,1 et $0,6 \%$ selon les critères de consensus américanoeuropéen [3]. La maladie touche surtout les femmes (90\%) entre 30 et 40 ans [4]. Un SGS secondaire est retrouvé chez

*Correspondance : fouz1981@hotmail.com 
$30 \%$ des malades atteints de polyarthrite rhumatoïde, $10 \%$ des malades atteints de lupus érythémateux disséminé et environ $5 \%$ des malades ayant une sclérodermie [3].

\section{Pathogénèse}

Le SGS survient chez des individus ayant une prédisposition génétique à l'auto-immunité, marquée par la prévalence élevée des haplotypes HLA B8 et DR3 et on retrouve une grande fréquence des maladies auto-immunes chez les apparentés des patients atteints d'un SGS (20 à $30 \%$ ) [1]. Sur ce terrain, une lésion des cellules épithéliales des glandes exocrines, secondaire par exemple à une infection virale, induirait le développement d'une inflammation chronique, caractérisée par un infiltrat lymphocytaire constitué de lymphocytes $T$ et lymphocytes B, avec formation de centres germinatifs. Cette inflammation serait auto-entretenue et aboutirait à un dysfonctionnement de la glande, puis à sa destruction [1].

Une étiologie virale a été suspectée dans le SGS comme dans toutes les maladies auto-immunes et plus encore dans ce cas du fait de la fréquence du portage viral dans la cavité buccale. Lors de la dernière décennie, l'attention s'est focalisée sur trois virus, le virus d'Epstein-Barr (EBV), les rétrovirus et le virus de l'hépatite $C(\mathrm{HCV})$, sans que des conclusions définitives sur leur responsabilité puissent être établies [3].

Trois hypothèses physiopathogéniques peuvent être envisagées [5] :

- les glandes salivaires sont une espèce de « poubelle » où l'on retrouve beaucoup de virus à l'état latent, ces virus sialotropes pouvant entraîner un syndrome sec sans autoanticorps, différent du SGS auto-immun ;

- le SGS peut être considéré comme un vrai syndrome avec différentes étiologies dont les virus sialotropes qui détermineraient un phénotype un peu particulier de la maladie ;

- les virus pourraient avoir un rôle plus indirect : tout virus spécifique d'une cible qui était génétiquement prédisposée à développer une maladie auto-immune entraîne des modifications de cette cible favorisant l'apparition de la maladie auto-immune.

Comme pour l'ensemble des maladies auto-immunes, l'origine du SGS est inconnue. Le primum movens de la maladie est probablement une anomalie des cellules épithéliales qui acquièrent toutes les caractéristiques pour attirer les cellules du système immunitaire. Ainsi, tous les épithélium pourraient être théoriquement atteints, d'où le nom d'épithélite autoimmune proposé par Moutsopoulos [6]. Cependant, le processus pathologique est généralement plus marqué dans les glandes salivaires et lacrymales, dont l'infiltration progressive par des cellules mononucléées entraine une sclérose parfois complète.

Les anomalies des cellules épithéliales seraient responsables de l'activité anormale des cellules lymphoïdes; on distingue deux types de troubles immunitaires. Le premier serait une activité anormale de cellules du système immunitaire comme le montrent les infiltrats inflammatoires de cellules mononucléées trouvés dans les glandes salivaires des malades. Ces infiltrats sont surtout constitués de lymphocytes T activés, et parfois aussi de lymphocytes B activés. Ces infiltrats sont à l'origine des principaux signes cliniques du SGS, en particulier de la sécheresse des muqueuses et des conjonctives, de la néphrite interstitielle, de la pneumonie interstitielle et de la méningo-encéphalite. Le second type de trouble immunitaire est l'activation oligoclonale des lymphocytes $B$. Il en résulte une hypergammaglobulinémie, des pics oligoclonaux à l'électrophorèse des protéines, et la production d'auto-anticorps. Parmi ceux-ci, on note le facteur rhumatoïde, les anti-SSA (anti-Ro) et les anti-SSB (anti-La) [3].

\section{Mécanismes à l'origine de la sécheresse des muqueuses}

La destruction des glandes ne peut expliquer à elle seule l'altération fonctionnelle (sécheresse) observée chez les patients. Au début de la maladie, l'infiltrat lymphocytaire est focal et une partie des glandes encore intacte. De plus, il n'y a pas de lien entre l'infiltrat lymphocytaire et la modification du flux salivaire. Il y a probablement une dysfonction des glandes restantes qui contribue à la gêne fonctionnelle; cette dysfonction serait due à une anomalie du contrôle de la sécrétion lacrymale et salivaire par le système nerveux autonome qui pourrait être liée à des auto-anticorps dirigés contre les récepteurs muscariniques $M 3$ [7, 8]. Il existe également un défaut d'expression membranaire de l'aquaporine 5, une protéine canal qui contrôle la sécrétion aqueuse des glandes lacrymales et salivaires [9] et dont l'expression est modulée par les récepteurs M3 [10]. Au total, la sécheresse serait due à la destruction glandulaire, mais aussi à un déficit du système nerveux autonome et à un défaut de l'expression membranaire de l'aquaporine 5. Ces données physiopathologiques ouvrent des perspectives intéressantes pour le traitement du SGS [11].

\section{Manifestations cliniques}

L'atteinte oculaire et buccale domine la symptomatologie par leur fréquence ; elle est retrouvée dans $90 \%$ des SGS [12]. Les signes buccaux sont ceux de la xérostomie qui représente le symptôme principal de l'atteinte des glandes salivaires. Elle se traduit par des troubles subjectifs et des altérations dans la cavité buccale révélées par l'examen clinique. Le patient se plaint de difficultés pour avaler, d'un manque de stabilité de ses prothèses dentaires amovibles, de l'apparition de caries, de brûlures chroniques de la muqueuse buccale, d'une intolérance aux aliments épicés ou acides, d'une incapacité à manger des aliments secs ou de parler de manière continue pendant 


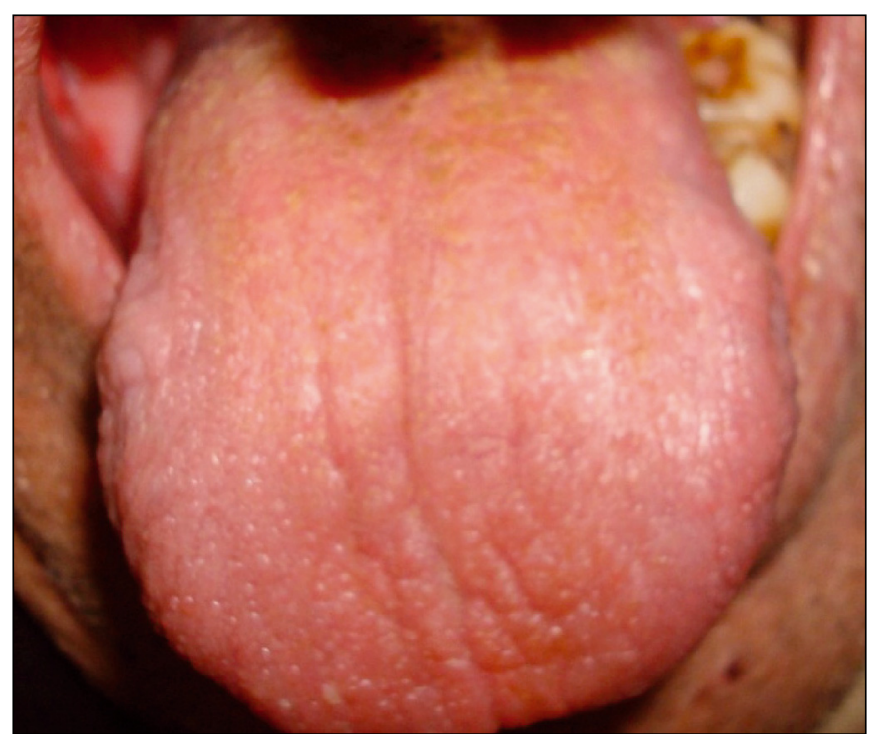

Fig. 1. Langue séche, partiellement dépapillée et fissuraire. Fig. 1. Dry, partially depapillated and fissurated tongue.

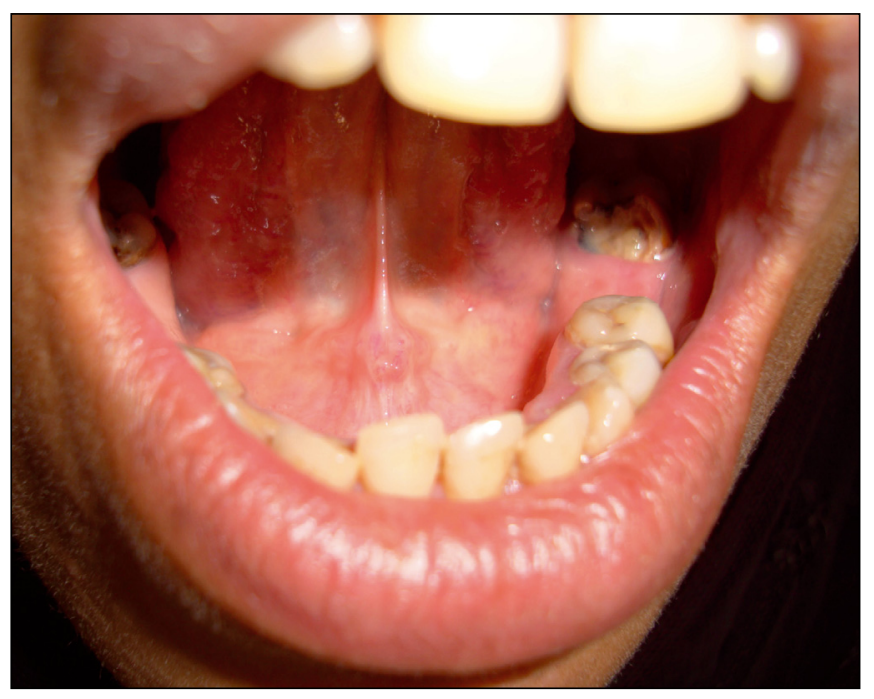

Fig. 2. Absence de lac salivaire sur le plancher buccal antérieur. Fig. 2. Lack of saliva on the anterior mouth floor.

quelques minutes. Le début des symptômes est insidieux et l'évolution se fait lentement. Le patient peut présenter une nycturie due à une augmentation des apports liquidiens $[2,13$, 14] et un reflux gastro-oesophagien en raison d'une évacuation ralentie de l'acide gastrique et la diminution de l'effet tampon de la salive [15]. À l'examen clinique, la xérostomie se traduit par une atteinte des tissus mous, des tissus durs et des glandes salivaires. La muqueuse buccale devient mate, collante et fragile. La langue est rouge, dépapillée et fissurée (Fig. 1), le lac de salive habituellement présent sur le plancher buccal est souvent inexistant (Fig. 2), un écoulement absent ou peu abondant de salive opaque à l'ostium des canaux des glandes salivaires

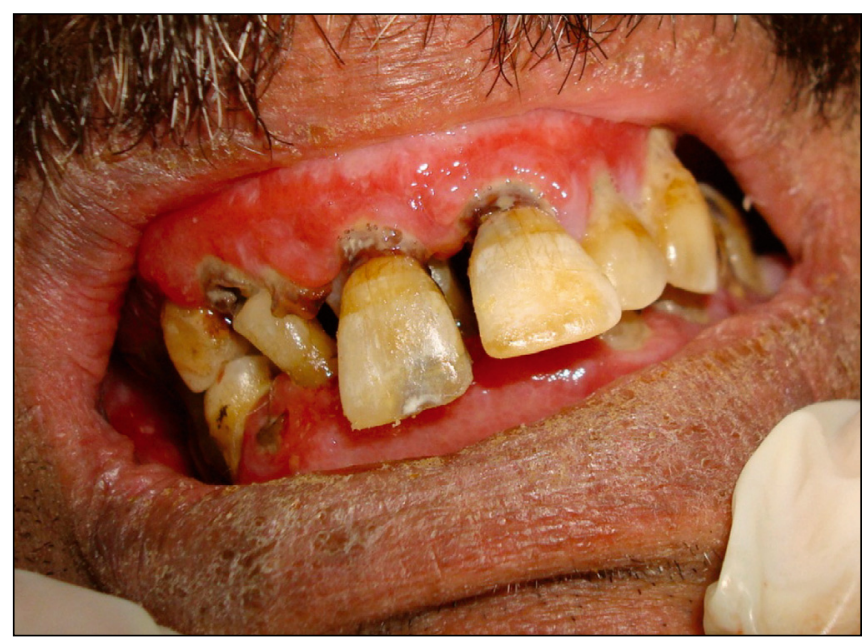

Fig. 3. Candidose érythémateuse. Fig. 3. Erythematous candidiasis.

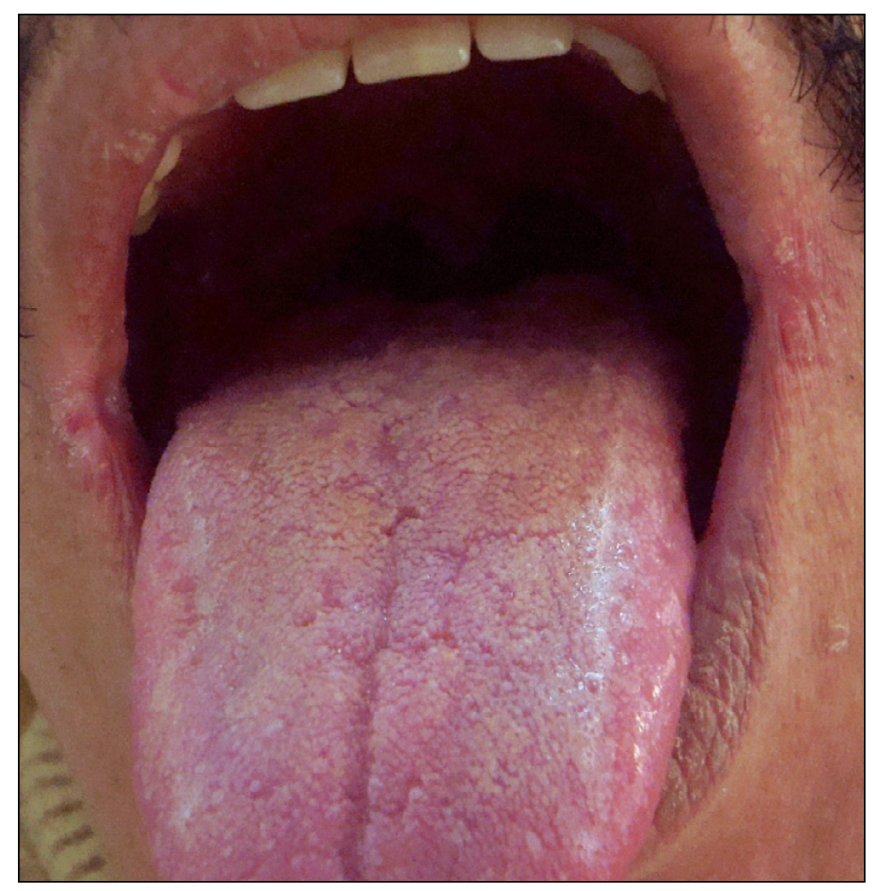

Fig. 4. Xérostomie avec perlèche. Fig. 4. Xerostomia and angular cheilitis.

principales $[13,14]$. L'infection candidosique est très fréquente dans le SGS ; elle pourrait atteindre $70 \%$ des patients [16] et se présente sous différentes formes : candidose pseudomembraneuse aiguë (muguet), candidose érythémateuse (Fig. 3), chéilite angulaire (perlèche) (Fig. 4), stomatite sousprothétique (Fig. 5). La prévention de l'infection candidosique est basée essentiellement sur une hygiène bucco-dentaire et prothétique adéquate [14]. Les malades atteints d'un SGS présentent un risque carieux élevé. Ces caries apparaissent sur 


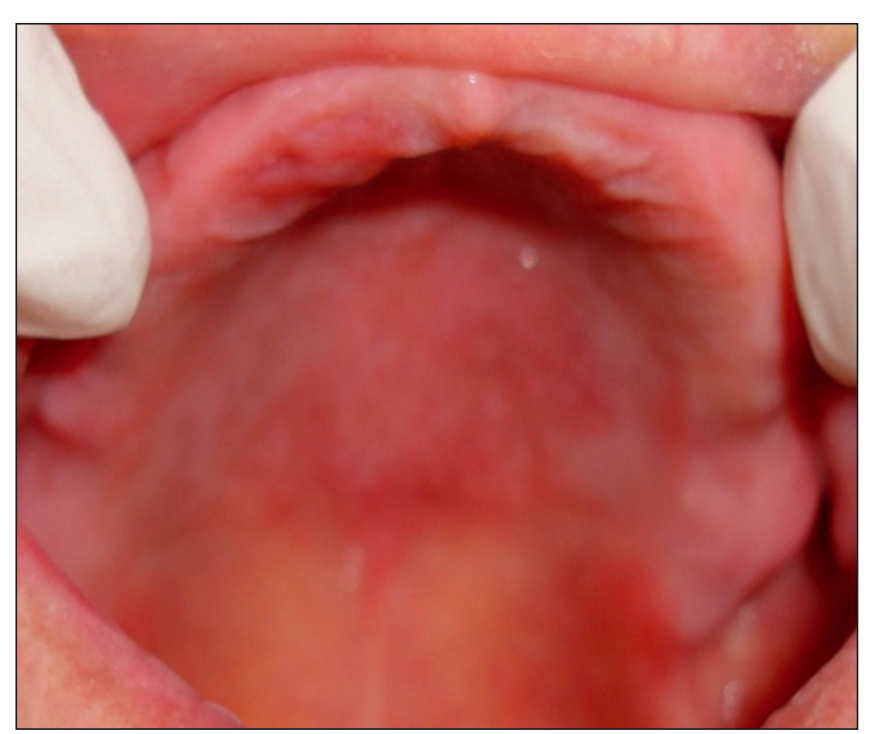

Fig. 5. Candidose sous-prothétique. Fig. 5. Denture stomatitis.

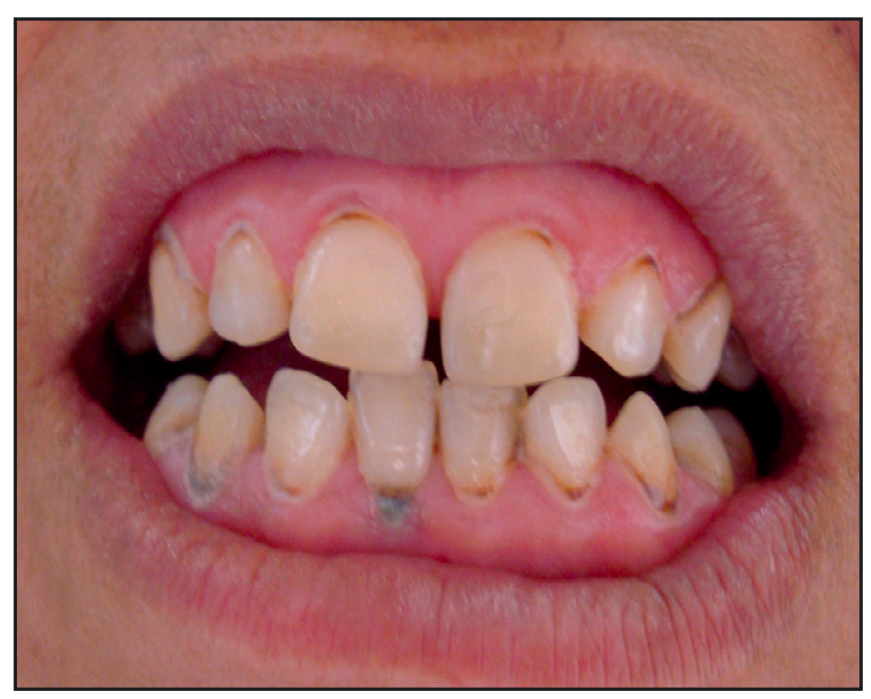

Fig. 6. Xérostomie, muqueuse buccale mate, collante et fragile avec présence de caries cervicales caractéristiques.

Fig. 6. Xerostomia, matt, sticky and fragile mucosa, and multiple caries in cervical location.

des sites habituellement assez protégés (dents mandibulaires antérieures, région cervicale, limites d'obturations récentes). Les caries cervicales sont prédominantes et elles ressemblent à celles rencontrées chez les patients ayant eu une irradiation cervico-faciale (Fig. 6) [14]. Il est recommandé chez ces malades de choisir des matériaux de restauration bioactifs qui relarguent du fluor et qui présentent aussi la capacité d'être rechargés par le fluor amené par une source exogène. Environ un tiers des patients développe, au cours de la maladie, une tuméfaction des glandes salivaires; le risque augmente avec la sévérité de la dysfonction glandulaire et l'inflammation [17].

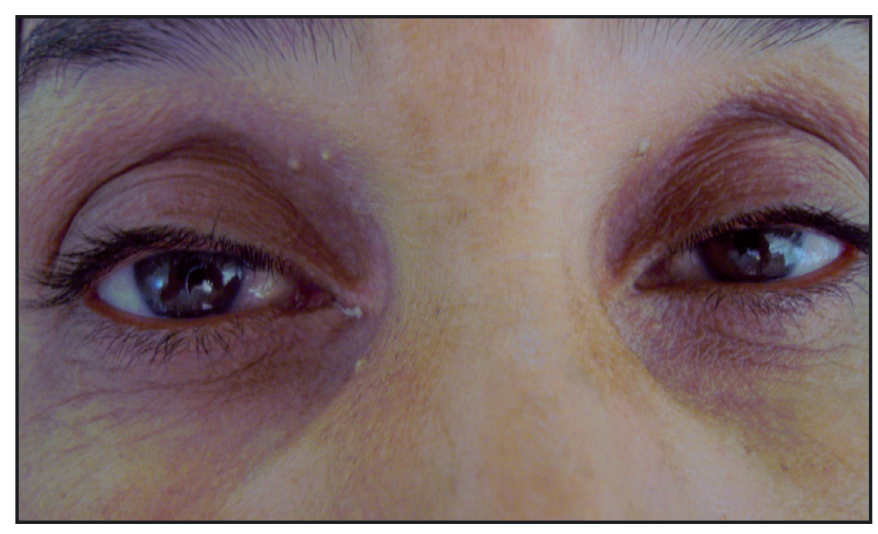

Fig. 7. Xérophtalmie.

Fig. 7. Dry eyes.

Les glandes salivaires peuvent présenter une tuméfaction le plus souvent bilatérale, diffuse, ferme et non douloureuse ou parfois légèrement sensible. Dans les formes précoces ou mineures, les glandes peuvent être discrètement indurées sans être tuméfiées. Les glandes submandibulaires sont parfois atteintes avant les glandes parotides. La tuméfaction peut être récurrente avec des épisodes évoluant sur quelques semaines ou quelques mois; dans d'autres cas, l'augmentation de volume est constante, avec peu ou pas de variation. Une sécrétion de couleur crème est parfois observée à l'ostium des canaux excréteurs; ce liquide contient des lymphocytes avec quelques polynucléaires neutrophiles. La surinfection bactérienne est peu fréquente, le patient présente alors les symptômes et les signes d'une sialadénite aiguë $[2,14,17]$. Pour les porteurs de prothèses dentaires amovibles, l'absence de salive diminue l'adhérence et la stabilité des prothèses. Leur port est souvent difficilement supportable. Les prothèses totales collent parfois aux lèvres, ce qui provoque des douleurs lors de leur pose et de leur dépose. La fragilité de la muqueuse explique la fréquence élevée des lésions sous-prothétiques [14].

Les signes fonctionnels oculaires correspondent à ceux $d^{\prime}$ une kératoconjonctivite sèche. Ce terme recouvre aussi bien la diminution de la production de larmes que les changements pathologiques touchant les cellules épithéliales de la surface de l'œil et des paupières. Les symptômes de la xérophtalmie (sensation de brûlures, sensation de corps étranger, photophobie, rougeur oculaire, et larmoiement réflexe s'il existe une atteinte cornéenne) varient d'un patient à l'autre car ils dépendent de la sévérité de la sécheresse oculaire (Fig. 7), de la capacité résiduel du film lacrymal à humidifier la surface oculaire et de la tolérance du patient à l'inconfort oculaire [12, 13].

Les manifestations générales sont nombreuses et de fréquence variable en fonction des différents appareils. Des manifestations articulaires sont présentes dans $50 \%$ des SGS primaires, un syndrome de Raynaud dans environ $30 \%$ des cas. On peut également observer des manifestations bronchopulmonaires (bronchite chronique secondaire à la sécheresse 
Tableau I. Critères diagnostiques du syndrome de SGS [18]. Table I. Diagnostic criteria for the Sjögren's syndrome.

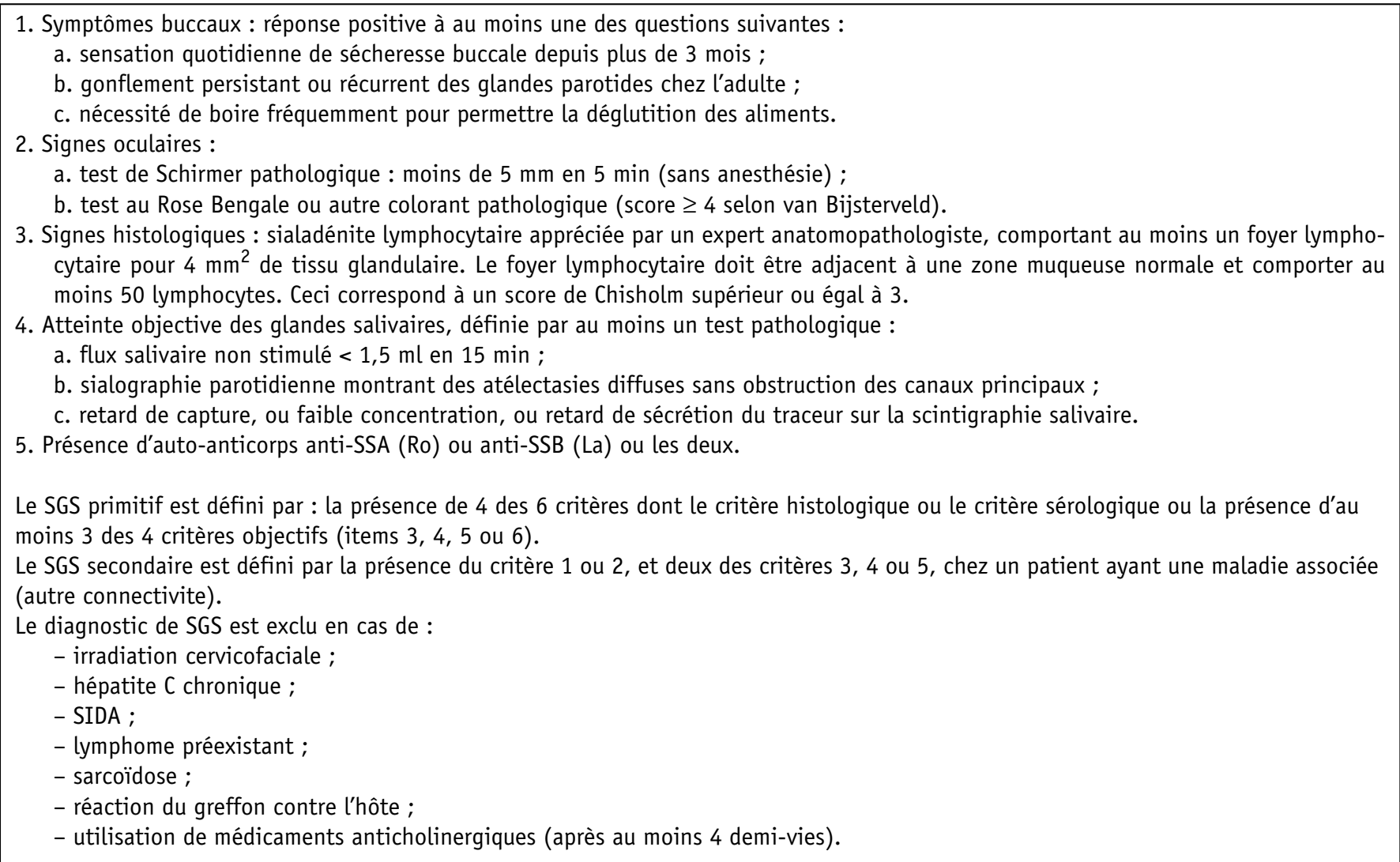

des voies aériennes supérieures ou à la fibrose pulmonaire interstitielle, associée à une hypertension artérielle pulmonaire), des manifestations gynécologiques, cutanées, digestives, neurologiques ou rénales en association avec le SGS [12]. Une surveillance hématologique est conseillée car $5 \%$ des patients atteints développent un lymphome non hodgkinien [3].

\section{Anomalies biologiques}

Le SGS s'accompagne fréquemment d'une hypergammaglobulinémie polyclonale, témoin de l'hyperactivité des lymphocytes B. Sur le plan immunologique, le facteur rhumatoïde est retrouvé dans $50 \%$ des formes débutantes de SGS ainsi que des taux élevés pour le test au latex et le test de Waaler-Rose. Les anticorps antinucléaires sont également retrouvés dans $50 \%$ des cas de SGS primitifs ou secondaires. Ces différents tests ne sont pas spécifiques du SGS. Par contre, les anticorps dirigés contre les antigènes solubles du noyau, l'anti-Ro (ou anti-SS-A) et l'anti-La (ou anti SS-B) présentent une bonne sensibilité et une bonne spécificité [12].

\section{Critères de diagnostic}

La définition du SGS a longtemps souffert de l'absence de critères diagnostiques précis et reconnus par tous les auteurs. Ceci est important car certains symptômes présentés par les malades (sécheresse buccale, fatigue, douleurs...) sont fréquemment rencontrés dans la population générale; ils peuvent être secondaires à la prise de médicaments, à un syndrome anxio-dépressif ou au vieillissement. Longtemps, il y a eu plusieurs définitions plus ou moins restrictives du SGS, d'où des prévalences très différentes. Depuis 2002, les critères européens modifiés se sont imposés (Tab. I) [18].

\section{SGS primitif}

Le diagnostic de SGS primitif nécessite l'existence d'une sécheresse buccale et oculaire ou la présence d'un infiltrat lymphocytaire sur une biopsie des glandes salivaires accessoires ou la présence d'auto-anticorps anti-SSA ou anti-SSB. Ces critères ont une spécificité de $96 \%$ et une sensibilité de $94 \%$ pour le diagnostic de SGS primitif [18]. 


\section{SGS secondaire}

Le SGS secondaire est défini par (1) la présence d'une autre connectivite, (2) l'existence d'une sécheresse oculaire ou buccale, et (3) un élément objectif de l'atteinte oculaire ou salivaire. La présence d'anticorps anti-SSA ou anti-SSB n'est pas nécessaire au diagnostic de SGS secondaire. Ces critères ont une spécificité de $97 \%$ et une sensibilité de $90 \%$ pour le diagnostic de SGS secondaire [18]. Parmi les connectivites, la polyarthrite rhumatoïde est la plus fréquemment associée au SGS secondaire, mais le lupus, la sclérodermie, la polymyosite ou la cirrhose biliaire primitive sont également rencontrés.

Selon ces critères, la présence d'une sarcoïdose ou d'une infection par le virus de l'hépatite $C$ exclut le diagnostic de SGS. Cependant, d'authentiques SGS peuvent exister dans ce contexte $[1,19,20]$.

\section{Traitement}

Le traitement est adapté à la sévérité des symptômes. Le tableau clinique est très variable allant d'un état asymptomatique à une forme très sévère. La plupart des patients ayant un SGS ne requièrent qu'un traitement local pour le syndrome sec. D'une manière générale, les médicaments pouvant aggravés le syndrome sec sont à éviter (rétinoïdes, bêtabloquants, antihistaminiques...). Les patients doivent apprendre à reconnaître et à éviter les endroits ayant une faible humidité (magasins et bureaux avec l'air conditionné), l'exposition au vent, les endroits poussiéreux, les substances irritatives et la fumée de cigarette.

Face à une xérostomie, il faut avant tout motiver les patients. L'hygiène bucco-dentaire doit être rigoureuse et régulière : brossage des dents après chaque repas avec une brosse à dents chirurgicale souple et un dentifrice fluoré, bains de bouche non agressifs (eau bicarbonatée), utilisation de jets dentaires et de fils parodontaux pour le nettoyage des espaces interdentaires, nettoyage et brossage systématique des prothèses amovibles qui seront déposées chaque nuit dans une solution antiseptique [14].

Le traitement topique de la xérostomie cherche à diminuer les symptômes de la sécheresse et prévenir les complications, c'est-à-dire les caries, la candidose ou les autres infections buccales, l'halitose et les lithiases salivaires. Ce traitement repose sur le remplacement de la sécrétion manquante et la stimulation de la sécrétion résiduelle. Le remplacement de la sécrétion manquante peut être facilement obtenu en buvant fréquemment des gorgées d'eau. Celle-ci ne doit pas forcément être avalée, elle peut être uniquement utilisée en rinçage. Cependant, l'eau n'humidifie et ne lubrifie pas de manière adéquate la muqueuse buccale et les dents [21]. Il existe plusieurs salives artificielles qui contiennent de la méthylcellulose ; certaines contiennent de la mucine d'origine animale pour diminuer la viscosité. Elles sont présentées sous différentes formes galéniques (liquide, pastille ou chewinggum). On trouve également dans le commerce d'autres substituts salivaires comme l'oxyde de polyéthylène [22] ou des extraits de polysaccharides provenant de graines de lin [23]. Le premier semble plus efficace que la méthylcellulose pour réduire les symptômes de xérostomie, le second donne des résultats satisfaisants chez environ $75 \%$ des patients. Des produits à base d'acide polyacrylique et de gomme de xanthan (« xanthan gum ») ont été développés et évalués chez des patients ayant un SGS [24]. Cette dernière étude a montré qu'il existe des sous-groupes de patients chez lesquels une forme particulière de substitut est plus efficace cliniquement. Les polymères hautement muco-adhésifs comme l'acide polyacrylique sont recommandés chez les patients ayant un flux salivaire extrêmement faible. Par contre, les patients qui ont encore un flux salivaire modéré ont plus d'amélioration avec les substituts ayant une adhérence muqueuse modéree et de bonnes propriétés élastiques (gomme de xanthan). En effet, ces polymères améliorent les propriétés physico-chimiques de la salive résiduelle, ce qui entraine une augmentation de l'humidification et de la lubrification.

La sécrétion salivaire résiduelle est stimulée par des stimulus mécaniques non spécifiques ou gustatifs. L'acide citrique, par exemple, provoque une stimulation gustative alors que la mastication du chewing-gum fait appel aux deux modalités. Chez les patients édentés, l'application d'un stimulus acide est limitée par la tolérance de la muqueuse. Chez les patients possédant encore des dents, la stimulation acide est déconseillée (risque de déminéralisation des dents). Lorsque la sécrétion salivaire résiduelle est assez élevée, la mastication de chewinggum sans sucre constitue un bon stimulus. Si elle est insuffisante, le chewing-gum colle aux dents. La succion de fruits séchés comme les pêches et les nectarines peut également stimuler la sécrétion salivaire. Avec la progression de la maladie, les stimulants deviennent insuffisants [25]. L'irrigation des glandes parotides avec une solution de corticostéroïdes permet d'obtenir une amélioration prolongée de la xérostomie, avec peu d'effets secondaires [26] mais il s'agit d'un traitement compliqué. Dans les 52 études randomisées et contrôlées, parues entre 1966 et 2001, sur le traitement de la xérostomie, Brennan et al. [27] n'ont trouvé que 4 études avec un niveau de preuves $A$, et ces 4 études confirmaient l'efficacité de la pilocarpine. Deux d'entre elles étaient spécifiquement dédiées aux patients ayant un SGS. Dans une étude préliminaire portant sur 5 patients, des bains de bouche à la cevimeline, 3 fois par jour, auraient entrainé une augmentation importante de la secrétion salivaire chez 2 patients et modérée chez les 3 autres [28].

Pour la réalisation de prothèses, les différentes étapes doivent tenir compte de la xérostomie. Lors du choix du matériau d'empreinte, plusieurs caractéristiques sont à prendre en considération : temps de prise, confort qu'il offre aux patients et surtout caractère hydrophile ou hydrophobe. Les 
bases prothétiques en résine acrylique sont plus facilement colonisées par le Candida albicans et moins rétentives que les bases métalliques [14]. Les salives artificielles en bains de bouche ou conditionnées en aérosols sont malheureusement d'une efficacité bréve (elle ne dépasse pas $30 \mathrm{~min}$ ). L'utilisation d'une prothèse réservoir permet de prolonger en bouche la présence de substituts salivaires ; $c^{\prime}$ est une prothèse amovible totale, comportant un réservoir que l'on remplit de salive artificielle à partir d'un orifice situé sur l'intrados prothétique. Un orifice situé sur l'extrados permet la libération de la salive au goutte-à-goutte par succion linguale. Le réservoir a un volume de 8 à $10 \mathrm{ml}$ qui correspond à une autonomie de $3-4 \mathrm{~h}$. Cette prothèse est mal tolérée en raison de son volume important $[29,30]$.

Seuls les médicaments agonistes des récepteurs muscariniques à action cholinergique augmentent réellement la secrétion salivaire, et dans une moindre mesure la secrétion lacrymale. Les principaux médicaments agonistes des récepteurs muscariniques sont le chlorhydrate de pilocarpine $\left(\right.$ Salagen $\left.{ }^{\circledR}\right)$ et la cevimeline $\left(\operatorname{Evoxac}^{\circledR}\right)[30,31]$. Le chlorhydrate de pilocarpine est prescrit à la dose d'une gélule à $5 \mathrm{mg} \times 4 \cdot \mathrm{j}^{-1}$ [32]. En cas d'inefficacité après 1 mois et de bonne tolérance, la dose peut être augmentée à $30 \mathrm{mg}^{-j^{-1}} \mathrm{en}$ 6 prises. Ce médicament à une durée d'action brève d'où la nécessité de multiplier les prises. Ce traitement peut entrainer des sueurs car toutes les glandes exocrines sont stimulées.

La cevimeline a été développée pour le traitement de la maladie d'Alzheimer car l'activité de l'agoniste M1 est neuroprotectrice. Elle semble plus spécifique du récepteur M3 salivaire. Sa demi-vie est plus longue, elle est donc généralement utilisée $\times 3 \cdot j^{-1}$, elle a montré son efficacité dans le syndrome $\mathrm{sec}$, avec une bonne tolérance clinique. Les principaux effets secondaires rapportés sont des céphalées, des sueurs et des douleurs abdominales [33].

Le traitement du syndrome sec oculaire comporte essentiellement des collyres substitutifs à type de larmes artificielles [34]. Les traitements généraux ne sont envisagés qu'en présence de manifestations systémiques. L'hydroxychloroquine aurait une certaine efficacité sur les arthralgies, les myalgies et l'asthénie. La corticothérapie et, a fortiori, les immunosuppresseurs ne sont prescrits qu'en cas de complications graves $[13,35]$.

\section{Conclusion}

Les manifestations buccales du SGS sont particulièrement fréquentes et mettent le médecin dentiste en position de poser le diagnostic. Ce diagnostic repose sur des éléments simples : un haut degré de suspicion clinique, la mise en évidence d'une sécheresse oculaire et buccale objective, la recherche d'autoanticorps anti-SSA et anti-SSB, et la réalisation d'une biopsie des glandes salivaires accessoires labiales inférieures. La confirmation du diagnostic entraine la mise en place de mesures de prévention et de surveillance, et le traitement des complications. Le médecin dentiste doit participer à la prise en charge du SGS, à la prévention en motivant le patient pour son hygiène bucco-dentaire et en surveillant l'évolution de la maladie et de ses complications.

Conflits d'intérêt : aucun

\section{Références}

1. Crestani B, Schneider S, Adle-Biassette H, Debray MP, Bonay M, Aubier M. Manifestations respiratoires au cours du syndrome de Gougerot-Sjögren. Rev Mal Respir 2007;24:535-51.

2. Sauvezie B, Deschaumes C, Rigal D, Baudet-Pommel M, Kemeny JL, Bonafous J, Prin P, Dubost JJ. Syndrome de Gougerot-Sjögren. Encycl Méd Chir Appareil locomoteur 14-223-A-10. Elsevier SAS, Paris, 2000.

3. Picone 0 , Alby C, Frydman R, Mariette X. Syndrome de GougerotSjögren en gynécologie obstétrique. Revue de la littérature. J Gynecol Obstet Biol Reprod 2006;35:169-75.

4. Kassan SS, Moutsopoulos HM. Clinical manifestations and early diagnosis of Sjögren syndrome. Arch Intern Med 2004;164: 1275-84.

5. Mariette X. Pathophysiology of Sjögren's syndrome. Ann Med Intern 2003;154:157-68.

6. Moutsopoulos HM. Sjogren's syndrome: autoimmune epithelitis. Clin Immunol Immunopathol 1994;72:162-5.

7. Fox RI. Sjogren's syndrome. Lancet 2005;366:321-31.

8. Dawson LJ, Stanbury J, Venn N, Hasdimir B, Rogers SN, Smith PM. Antimuscarinic antibodies in primary Sjogren's syndrome reversibly inhibit the mechanism of fluid secretion by human submandibular salivary acinar cells. Arthritis Rheum 2006; 54:1165-73.

9. Steinfeld S, Cogan E, King LS, Agre P, Kiss R, Delporte C. Abnormal distribution of aquaporin-5 water channel protein in salivary glands from Sjogren's syndrome patients. Lab Invest 2001; 81:143-8.

10. Li J, Ha YM, Ku NY, Choi SY, Lee SJ, Oh SB, Kim JS, Lee JH, Lee $E B$, Song YW, Park K. Inhibitory effects of autoantibodies on the muscarinic receptors in Sjogren's syndrome. Lab Invest 2004; 84:1430-8.

11. Fox RI. Sjogren's syndrome: evolving therapies. Expert Opin Investig Drugs 2003;12:247-54.

12. Pisella PJ, Creuzot-Garcher C, Baudouin C. Actualités sur le syndrome de Gougerot-Sjögren. J Fr Ophtalmol 1999;22:889-92.

13. Gentric-Tilly A. Syndrome de Gougerot-Sjögren du sujet âgé. Ann Med Interne 2002; 153:378-82.

14. Baudet-Pommel M, Deschaumes C, Sauvezie D, Sauvezie B. Syndrome de Gougerot-Sjogren : complications bucco-dentaires. Actual Odonto-Stomatol 2001;213:65-77.

15. Korsten MA, Rosman AS, Fishbein S, Shlein RD, Goldberg HE, Biener A. Chronic xerostomia increases esophageal acid exposure and is associated with esophageal injury. Am J Med 1991;90:701-6.

16. Piette $\mathrm{E}$, Reychler $\mathrm{H}$. Traité de pathologies buccales et maxillofaciales (pp. 1116-8). De Boeck Université, Bruxelles, 1991.

17. Daniels TE. Labial salivary gland biopsy in Sjögren's syndrome: assessment as a diagnostic criterion in 362 suspected cases. Arthritis Rheum 1984;27:147-56. 
18. Vitali C, Bombardieri S, Jonsson R, Moutsopoulos HM, Alexander EL, Carsons SE, Daniels TE, Fox PC, Fox RI, Kassan SS, Pillemer SR, Talal N, Weisman MH. Classification criteria for Sjogren's syndrome: a revised version of the European criteria proposed by the American-European consensus group. Ann Rheum Dis 2002;61:554-8.

19. Ramos-Casals M, Loustaud-Ratti V, De Vita S, Zeher M, Bosch JA, Toussirot E, Medina F, Rosas J, Anaya JM, Font J. Sjogren syndrome associated with hepatitis $($ virus: a multicenter analysis of 137 cases. Medicine (Baltimore) 2005;84:81-9.

20. Ramos-Casals M, Brito-Zeron P, Garcia-Carrasco M, Font J. Sarcoidosis or Sjogren syndrome? Clues to defining mimicry or coexistence in 59 cases. Medicine (Baltimore) 2004;83:85-95.

21. Vissink A, De Jong HP, Busscher HJ, Arends J, Gravenmade EJ. Wetting properties of human saliva and saliva substitutes. J Dent Res 1986;65:1121-4.

22. Marks NJ, Roberts BJ. A proposed new method for the treatment of dry mouth. Ann R Coll Surg Engl 1983;65:191-3.

23. Johansson G, Andersson G, Attstrom R, Glantz P0, Larsson K. The effect of Salinum on the symptoms of dry mouth: a pilot study. Gerodontology 1994;11:46-9.

24. van der Reijden WA, van der Kwaak H, Vissink A, Veerman EC, Amerongen AV. Treatment of xerostomia with polymer-based saliva substitutes in patients with Sjogren's syndrome. Arthritis Rheum 1996;39:57-63.

25. Vissink A, Panders AK, Nauta JM, Ligeon EE, Nikkels PG, Kallenberg CG. Applicability of saliva as a diagnostic fluid in Sjogren's syndrome. Ann N Y Acad Sci 1993;694:325-9.
26. Izumi M, Eguchi K, Nakamura H, Takagi Y, Kawabe Y, Nakamura T. Corticosteroid irrigation of parotid gland for treatment of xerostomia in patients with Sjögren's syndrome. Ann Rheum Dis 1998;57:464-9.

27. Brennan MT, Shariff G, Lockhart PB, Fox PC. Treatment of xerostomia: a systematic review of therapeutic trials. Dent Clin North Am 2002;46:847-56.

28. Takagi $Y$, Kimura $Y$, Nakamura T. Cevimeline gargle for treatment of xerostomia in patients with Sjögren's syndrome. Ann Rheum Dis 2004;63:749.

29. Ragot J-P, Auriol M, Bertrand J-C. Vieillissement des glandes salivaires : bouches sèches des personnes âgées. Actual OdontoStomatol 2001;215:345-65.

30. Strietzel FP, Martin-Granizo R, Fedele S, Lo Russo L, Mignogna M, Reichart PA, Wolff A. Electrostimulating device in the management of xerostomia. Oral Dis 2007;13:206-13.

31. Iwabuchi Y, Masuhara T. Sialogogic activities of SNI-2011 compared with those of pilocarpine and McN-A-343 in rat salivary glands: identification of a potential therapeutic agent for treatment of Sjorgen's syndrome. Gen Pharmacol 1994;25:123-9.

32. Mariette $X$. Les syndromes secs médicamenteux. Rev Rhum 2002;69:386-9.

33. Fife RS, Chase WF, Dore RK, Wiesenhutter CW, Lockhart PB, Tindall $E$, Suen JY. Cevimeline for the treatment of xerostomia in patients with Sjogren syndrome: a randomized trial. Arch Intern Med 2002;162:1293-300.

34. Roncin S. L'œil sec. Rev Prat 2001;31:140-7.

35. Hatron PY, Fauchais A. Le syndrome de Gougerot-Sjögren primitif. Rev Prat 2001;51:159-64. 